\title{
Práticas ambientais sob a perspectiva da tecnologia social
}

\section{RESUMO}

Sérgio Batista Oliveira

batistaoliveiras@yahoo.com.br Universidade Tecnológica Federal do Paraná- campus Cornélio Procópio Universidade Estadual do Norte do Paraná - campus de Cornélio Procópio

Odair Aquino Campos

aquinocampos@terra.com.br

Escola Técnica Jacinto Ferreira de Sá Ourinhos/SP

Carlos Cesar Garcia Freitas

cesarfreitas@uenp.edu.br

Programa de Pós-Graduação em Ensino

Universidade Estadual do Norte do

Paraná - campus de Cornélio Procópio

João Coelho Neto

joaocoelho@uenp.edu.br

Programa de Pós-Graduação em Ensino Universidade Estadual do Norte do Paraná - campus de Cornélio Procópio
A Tecnologia Social tem contribuído na tentativa de minimizar muitos dos problemas sociais não atendidos pela esfera pública, de modo especial relacionados com a problemática do Meio Ambiente. Dessa forma, este artigo visou analisar os projetos de Tecnologias Sociais que apresentam soluções para as questões ambientais. Para tanto, como encaminhamento metodológico foi utilizada uma abordagem de pesquisa qualitativa e os dados colhidos no banco de Tecnologias Sociais da Fundação Banco do Brasil. Como resultado foram identificados 78 projetos de um total de 1011 registrados no banco de dados analisados; essas tecnologias podem auxiliar na emancipação das comunidades que são beneficiadas pela sua implementação, promovendo a inclusão social, diminuição da fronteira entre as classes sociais e melhorando a qualidade de vida dos envolvidos. Ainda, foi constatado que os anos de 2008 e 2009 foram os períodos de maior implantação de projetos e o principal público consistiu em agricultores.

PALAVRAS-CHAVE: Meio Ambiente. Tecnologias Sociais. Alternativas Tecnológicas. 


\section{INTRODUÇÃO}

De acordo com o Anexo I, da Resolução 306/2002 do Conselho Nacional do Meio Ambiente (CONAMA), Meio Ambiente é o "[...] conjunto de condições, leis, influência e interações de ordem física, química, biológica, social, cultural e urbanística, que permite, abriga e rege a vida em todas as suas formas" (CONAMA, 2002 , p. 760 ), assim, o ser humano interage com outras formas de vida e viceversa.

Devido essa interação, a questão ambiental assumiu uma posição de destaque nas discussões sobre os problemas sociais devido à percepção de seu impacto no dia a dia das pessoas. Inundações, secas, aumento da temperatura, desmatamento, erosão do solo, poluição dos rios, entre outros acontecimentos tem ocorrido com maior frequência.

Desse modo, surge o conceito do impacto ambiental que segundo a Resolução 306/2002 do CONAMA é:

[...] qualquer alteração das propriedades físicas, químicas e biológicas do meio ambiente, causada por qualquer forma de matéria ou energia resultante das atividades humanas que, direta ou indiretamente, afetam a saúde, a segurança e o bemestar da população, as atividades sociais e econômicas, a biota, as condições estéticas e sanitárias do meio ambiente e a qualidade dos recursos ambientais (CONAMA, 2002, p. 759760).

Portanto, as atividades humanas interferem no Meio Ambiente, sendo muitas delas por meio da utilização de tecnologias que podem trazer consequências desagradáveis ao Meio Ambiente e ao próprio homem, pois "[...] o Homem faz parte da Natureza, e assim devemos enxergá-lo. Homem e Natureza são indissociáveis [...]" (OLIVEIRA, 2010, p. 44).

Segundo Gapinski et al. (2018), as várias dimensões que estão presentes no desenvolvimento sustentável podem ser atendidas pela Tecnologia Social, a partir do uso de tecnologias alternativas, voltadas para a transformação social, ligadas às demandas da sustentabilidade social, econômica e ambiental.

A forma de usar as tecnologias muitas vezes desequilibra a relação do homem com a natureza, ocasionando consequências indesejáveis, como: a poluição do ar pelas indústrias e automóveis; uso de agrotóxico com a contaminação do solo e dos rios; dentre muitas outras formas de impacto ambiental que podem ser provocadas pelo emprego de aparatos tecnológicos.

Nesse sentido, Garcia (2014) diz que a Tecnologia Social (TS) pode exercer um papel fundamental no que diz respeito a questão da sustentabilidade, seja econômica, social, política, cultural e ambiental. A TS, como relata Costa (2013), é um conjunto de técnicas, metodologias transformadoras, desenvolvidas e que podem ser aplicadas na interação e na apropriação com a população, que representam soluções para inclusão social e melhoria das condições de vida.

No mesmo sentido, explica De Magalhães et al. (2008) que a TS tem como base de sua essência, pesquisas, conhecimentos populares ou científicos e tecnológicos, a qual tem como objetivo solucionar os problemas enfrentados por uma população, seja para suas necessidades básicas, como alimentação, saúde e saneamento, até para atividades mais elaboradas, como de desenvolvimento, 
defesa do Meio Ambiente, entre outras, como pode ser observado no caso da área da Tecnologia Assistiva, a qual possibilita que pessoas com alguma necessidade possa ter autonomia.

Diante do exposto surge a indagação: de que modo a Tecnologia Social tem se apresentado como solução para a problemática ambiental?

A questão levantada, permite formular o seguinte objetivo de pesquisa: identificar as Tecnologias Sociais que abordem a temática Meio Ambiente em seu contexto.

Para compor esse objetivo, foi desenvolvida uma pesquisa exploratória qualitativa das Tecnologias Sociais junto ao banco de dados de tecnologias sociais do Banco do Brasil que abordavam a questão do Meio Ambiente e a relação com os atores envolvidos.

Para esclarecimentos dos leitores, este artigo está estruturado nas partes que segue: na seção um, contextualiza a temática; na seção dois, apresenta referencial teórico; na seção três, material e métodos; na seção quatro, resultados e discussões e na seção cinco, as considerações finais.

\section{REFERENCIAL TEÓRICO}

Nessa seção, apresenta-se o aporte teórico sobre o Meio Ambiente e a Tecnologia Social, como base teórica para esta pesquisa.

Meio Ambiente: contextos e aproximações

Com o advento da industrialização e do capitalismo, a relação do homem com - Meio Ambiente mudou drasticamente em função do desenfreado comportamento de consumo da sociedade "moderna", e "calorosos" debates têm questionado os "progressos", uma vez que o "atual modelo de desenvolvimento é caracterizado pela exploração dos recursos naturais e o consumo excessivo destes" (REIS, 2012 p.7).

Como mecanismo de manutenção e alavancagem desse desenvolvimento a tecnologia tem sido utilizada de modo cada vez mais intensa pelo homem, contudo, o uso acrítico, baseado em uma orientação meramente mercadológica, tem gerado consequências desagradáveis não somente ao Meio Ambiente, mas ao próprio homem que depende deste. Cabe destacar que "[...] o Homem faz parte da Natureza, e assim devemos enxergá-lo. Homem e Natureza são indissociáveis [...]" (OLIVEIRA, 2010, p. 44) em uma relação de dependência.

Portanto, o homem ao fazer uso da tecnologia não pode se imaginar fora da natureza, a despeito de viver, não raras vezes, como se suas ações não tivessem relação direta com o meio-ambiente, como destaca Feenberg (2009, p. 109):

Quando agimos tecnologicamente na natureza, imaginamo-nos fora do sistema ecológico, mas, na realidade, isso inclui-nos assim como os objetos da nossa ação [...]. Quem dirige automóvel chega ao seu destino aparentemente sem mal nenhum, entretanto, o ar acima deles se enche com a poluição que causa problemas respiratórios para todos, incluindo a si e a sua família [...]. 
Na realidade, a tecnologia foi criada pelo homem para mudar o mundo e o impacto ambiental de seu uso, quando de modo inadequado, pode trazer sérios prejuízos a esse, sendo suas consequências perceptíveis em dado espaço e tempo.

[...] No caso da poluição, tudo o que é preciso fazer para identificar a reação é ampliar o contexto [...]. Com efeito, todos os venenos produzidos pela indústria acabam no quintal de alguém, mesmo que leve anos para perceber. À medida que a tecnologia se torna mais poderosa, seus efeitos secundários negativos se tornam mais difíceis de ignorar e, finalmente, é impossível silenciar aqueles que sofrem esses efeitos secundários (FEENBERG, 2009, p. 110).

À medida que a tecnologia se torna mais poderosa, o homem ao fazer uso dela, por vezes esquece ou não percebe o impacto que seu ato faz na natureza. Assim, qualquer alteração que o homem realiza no Meio Ambiente retorna de alguma maneira em um determinado tempo e lugar.

Desse modo, há a necessidade de uma visão mais ampla do homem e de sua relação com o Meio Ambiente, isto é, uma abordagem multidisciplinar entre Ciências Naturais e do comportamento humano (REIS, 2012), o que implica o imperativo da busca de alternativas tecnológicas que restabeleçam uma relação saudável entre o homem (utilização dos recursos naturais para sobreviver e se manter) e o ambiente (preservação), entre essas, destaca-se a Tecnologia Social, o qual será explanada na próxima subseção.

\section{Tecnologia Social}

Segundo Duque e Valadão (2017), a terminologia Tecnologia Social apesar de ser empregada apenas recentemente a partir de 2004, tem-se registros históricos, datados das décadas de 1970 e 1980, da existência de práticas caracterizadas como próximas das conceituações atuais, baseadas no uso de tecnologias em pequena escala, que eram voltadas para o uso familiar ou comunitárias e tinham um baixo custo na sua produção.

Utilizada em diversas aplicações, Pereira e Freitas (2018) discorrem que a Tecnologia Social pode ser classificada de três formas: fenômeno de emancipação social, de resgate de valores e de um processo educacional. Em todas elas o conhecimento é elemento central e sua difusão é imperativo para que a transformação do sujeito possa ocorrer; é por meio da apropriação (posse e conhecimento) da Tecnologia Social que se dá a capacidade do indivíduo mudar sua realidade, da sua comunidade ou até mesmo de uma cidade.

O fenômeno Tecnologia Social é definido pela Rede de Tecnologia Social como "um conjunto de técnicas, metodologias transformadoras, desenvolvidas e/ou aplicadas na interação com a população e apropriadas por ela, que representam soluções para inclusão social e melhoria das condições de vida" (COSTA, 2013, p. 26).

Entre suas características, evidencia-se a preocupação com a participação efetiva da comunidade no seu processo de construção ou apropriação e são "[...] diversas as suas aplicações, como: empreendedorismo social, empreendedorismo 
solidário, inovação social, incubadoras sociais, economia solidária" (LUNARDI, 2015, p. 61).

Além de suas aplicações, Valadão, Andrade e Cordeiro Neto (2014) discorrem que a Tecnologia Social pode ser vista como um novo conceito capaz de superar o determinismo tecnológico, e com isso, criar condições para que uma nova visão integre as questões técnicas e sociais voltadas para a análise da tecnologia na sociedade, por meio da integração entre o social e o técnico.

Nesta perspectiva, os projetos de Tecnologia Social constituem-se em alternativas tecnológicas inovadoras, seja pela sua dimensão de processo social, como pelos resultados que proporcionam em termos de melhoria na qualidade de vida (BAVA, 2004).

Além disso, são soluções construídas e orientadas pelas necessidades das pessoas ou comunidades. São criadas e desenvolvidas com a finalidade de oferecer a essas, meios para que de modo próprio possam solucionar seus problemas, daí a preocupação com o processo de emancipação dos indivíduos.

De um modo coletivo, a Tecnologia Social foi pensada como política pública capaz de possibilitar a melhoria na qualidade de vida das pessoas, assim, contribuindo para a redução da pobreza e um Meio Ambiente mais equilibrado (WEISS, 2009).

Desse modo, a Tecnologia Social visa em seus princípios e parâmetros a valorização de soluções que permitam colocar a tecnologia a serviço do homem e, para tanto, apresentam características como: ser adaptável; ter o conhecimento democratizado; satisfazer as necessidades humanas; incentivar e potencializar a criatividade de seus usuários; possibilitar a viabilidade econômica, ambiental e social, entre outras (DAGNINO; NOVAES, 2004).

Antes de implantar a Tecnologia Social em qualquer contexto, deve-se sempre considerar as realidades sociais locais, de modo a respeitar os valores ali presentes. Ainda, empregar-se preferencialmente as formas de organização coletiva e processos democráticos de tomada de decisão (LASSANCE JR.; PEDREIRA, 2004 apud BAUMGARTEN, 2006).

Apesar da denominação muitas vezes passar despercebida, as Tecnologias Sociais estão espalhadas por várias áreas como: Saúde, Educação, Renda, Agricultura, entre outras, chegando as pessoas do sul ao norte do Brasil (LASSANCE JR; PEDREIRA, 2004) e tem contribuído para minimizar muitos dos problemas sociais não atendidos pela esfera pública, de modo especial relacionados com a problemática do Meio Ambiente.

Nessa seção, explanou-se um pouco sobre a Problemática Social e a Tecnologia Social, permitindo que na próxima seção seja apresentada a Metodologia utilizada nesta pesquisa. 


\section{METODOLOGIA}

No desenvolvimento dessa pesquisa, utilizou-se a pesquisa exploratória qualitativa, que conforme Gil (2010), tem como objetivo proporcionar maior familiaridade com o tema proposto, a fim de torná-lo mais explícito ou para construir hipóteses.

Assim, para a realização dessa pesquisa foram utilizadas algumas etapas, as quais estão descritas a seguir.

1. Foi realizado um levantamento das Tecnologias Sociais junto ao banco de dados de Tecnologias Sociais do Banco do Brasil, que tinha como tema principal ou secundário a questão do Meio Ambiente. Esse levantamento aconteceu no período de agosto a dezembro de 2017, podendo haver alterações em pesquisas posteriores.

2. Após esse levantamento organizou-se os dados da pesquisa em uma planilha de classificação dos dados, assim, classificando-os em temáticas.

3. Em seguida, após o levantamento desses dados, foi feita uma análise, por meio de tabelas e gráficos para identificar quais as regiões, público alvo, valor gasto para implementação das Tecnologias Sociais, quantidade de reaplicações, ano da sua certificação, entre outros pontos.

4. Posteriormente foram feitas as análises em separado de cada gráfico e tabela, para poder mapear de forma sistemática de que modo a Tecnologia Social tem se apresentado como solução para a problemática ambiental.

5. O fato da escolha do banco de dados de Tecnologias Social do Banco do Brasil, deve-se as Tecnologias Sociais registradas terem sido reconhecidas e certificadas, o que possibilita a validade dos dados coletados na pesquisa. Afinal, para que uma tecnologia seja classificada como Tecnologia Social são levadas em consideração, vários critérios como: o envolvimento da população local, disseminação para os demais membros das comunidades envolvidas, da qualidade de vida da população local, entre outros.

A próxima seção, traz os Resultados e Discussões acerca do mapeamento realizado.

\section{RESULTADOS E DISCUSSÕES}

Junto ao universo de 1011 Tecnologias Sociais analisadas do banco de Tecnologias Sociais do Banco do Brasil, foram identificados 78 projetos que trabalham a questão do Meio Ambiente, como tema principal ou secundário.

Com a Tabela 1, foi possível identificar, o ano de implantação das Tecnologias Sociais e o número de reaplicação que tiveram os projetos. 
Tabela 1 - Ano de Implantação e número de Reaplicações das Tecnologias Sociais

\begin{tabular}{|c|c|c|c|c|}
\hline $\begin{array}{c}\text { Ano da } \\
\text { Implantação }\end{array}$ & $\begin{array}{c}\text { Quantidade } \\
\text { implantada } \\
\text { por ano }\end{array}$ & $\begin{array}{c}\text { \% do número } \\
\text { de } \\
\text { Implantação }\end{array}$ & $\begin{array}{l}\text { Número de } \\
\text { reaplicação }\end{array}$ & $\begin{array}{c}\text { \% do número } \\
\text { de } \\
\text { reaplicação }\end{array}$ \\
\hline 1969 & 1 & $1,28 \%$ & 11 & $3,6 \%$ \\
\hline 1992 & 1 & $1,28 \%$ & 2 & $0,7 \%$ \\
\hline 1995 & 2 & $2,56 \%$ & 8 & $2,6 \%$ \\
\hline 1998 & 1 & $1,28 \%$ & 1 & $0,3 \%$ \\
\hline 1999 & 1 & $1,28 \%$ & 1 & $0,3 \%$ \\
\hline 2000 & 2 & $2,56 \%$ & 4 & $1,3 \%$ \\
\hline 2001 & 1 & $1,28 \%$ & 19 & $6,2 \%$ \\
\hline 2002 & 7 & $8,97 \%$ & 29 & $9,5 \%$ \\
\hline 2003 & 1 & $1,28 \%$ & 4 & $1,3 \%$ \\
\hline 2004 & 3 & $3,85 \%$ & 29 & $9,5 \%$ \\
\hline 2005 & 5 & $6,41 \%$ & 6 & $2,0 \%$ \\
\hline 2006 & 3 & $3,85 \%$ & 65 & $21,2 \%$ \\
\hline 2007 & 5 & $6,41 \%$ & 31 & $10,1 \%$ \\
\hline 2008 & 8 & $10,26 \%$ & 0 & $0,0 \%$ \\
\hline 2009 & 10 & $12,82 \%$ & 30 & $9,8 \%$ \\
\hline 2010 & 5 & $6,41 \%$ & 6 & $2,0 \%$ \\
\hline 2011 & 4 & $5,13 \%$ & 44 & $14,4 \%$ \\
\hline 2012 & 3 & $3,85 \%$ & 3 & $1,0 \%$ \\
\hline 2013 & 6 & $7,69 \%$ & 6 & $2,0 \%$ \\
\hline 2014 & 2 & $2,56 \%$ & 6 & $2,0 \%$ \\
\hline 2015 & 3 & $3,85 \%$ & 1 & $0,3 \%$ \\
\hline 2016 & 1 & $1,28 \%$ & 0 & $0,0 \%$ \\
\hline 2017 & 2 & $2,56 \%$ & 0 & $0,0 \%$ \\
\hline $\begin{array}{c}\text { Não } \\
\text { Informado }\end{array}$ & 1 & $1,28 \%$ & 0 & $0,0 \%$ \\
\hline Total & 78 & $100 \%$ & 306 & $100 \%$ \\
\hline
\end{tabular}

Vale ressaltar que a Tabela 1, apresenta o ano da implementação das Tecnologias Sociais e a quantidade de reaplicação que essas tiveram no decorrer dos anos. Pode-se observar que o ano de 2006 foi o que teve a maior quantidade de reaplicação, 65 no total. $O$ objetivo principal dessas tecnologias foram a questão da preservação das nascentes de água, por meio do reflorestamento da mata ciliar.

Outro ponto, foi a redução da emissão de carbono, substituindo a lenha nativa por biomassa renovável nos fornos. Em seguida, tem-se o ano de 2011 com 44 reaplicações, que tratava de assuntos relacionados a integração da Educação e Cultura, que envolviam a questão ambiental na melhoria do ambiente escolar. Somando todas as reaplicações nesse período, chegou-se a uma quantidade total de 306.

Na Tabela 1, observou-se também que, a primeira Tecnologia Social, registrada junto ao banco de tecnologias do Banco do Brasil, foi criada em 1969. O ano de 2009, foi o ano que teve o maior número de tecnologias implementadas, com 10 no decorrer de todo o ano.

Na Tabela 2, é apresentado o valor estimado para a implantação das Tecnologias Sociais. 
Tabela 2 - Valor estimado para implantação das Tecnologias Sociais

\begin{tabular}{|c|c|c|}
\hline $\begin{array}{l}\text { Faixa de Valor Estimado para Implantação } \\
\text { da Tecnologia (R\$̦) }\end{array}$ & Quantidade & Percentual \\
\hline De 70,00 a $1.500,00$ & 9 & $11,5 \%$ \\
\hline De $1.500,00$ a $10.000,00$ & 15 & $19,2 \%$ \\
\hline De $10.000,00$ a $50.000,00$ & 16 & $20,5 \%$ \\
\hline De $50.000,00$ a $100.000,00$ & 14 & $17,9 \%$ \\
\hline De $100.000,00$ a $500.000,00$ & 14 & $17,9 \%$ \\
\hline De $500.000,00$ a $1.000 .000,00$ & 3 & $3,9 \%$ \\
\hline De $1.000 .000,00$ a $3.000 .000,00$ & 3 & $3,9 \%$ \\
\hline De $3.000 .000,00$ a $5.000 .000,00$ & 1 & $1,3 \%$ \\
\hline Não informado & 3 & $3,9 \%$ \\
\hline Total & 78 & $100 \%$ \\
\hline
\end{tabular}

Fonte: dados da pesquisa (2017)

Em relação aos investimentos, a tecnologia CONCEITO BASE ZERO-CBZ: MODO GERAL DE ORGANIZAR PRODUÇÃO RENOVÁVEL SUSTENTÁVEL, apresentou o maior valor, estimado em $\mathrm{R} \$ 5.000 .000,00$ ao longo de 46 anos e que proporcionou a emancipação de 3,6 mil de famílias ou 18 mil de pessoas. Foi a tecnologia que teve o maior investimento dentre as tecnologias cadastradas junto ao banco de Tecnologia Social do Banco do Brasil.

Em contrapartida, a que teve o menor valor para sua implementação foi a ABELHAS NATIVAS, com um valor de R\$ 70,00. É importante registrar que 2 das 78 Tecnologias Sociais analisadas não informaram o custo estimado para a sua implementação.

O Gráfico 1, traz a mensuração do número de projetos implantados por Estado brasileiro.

Gráfico 1 - Número de projetos implantados por Estado.

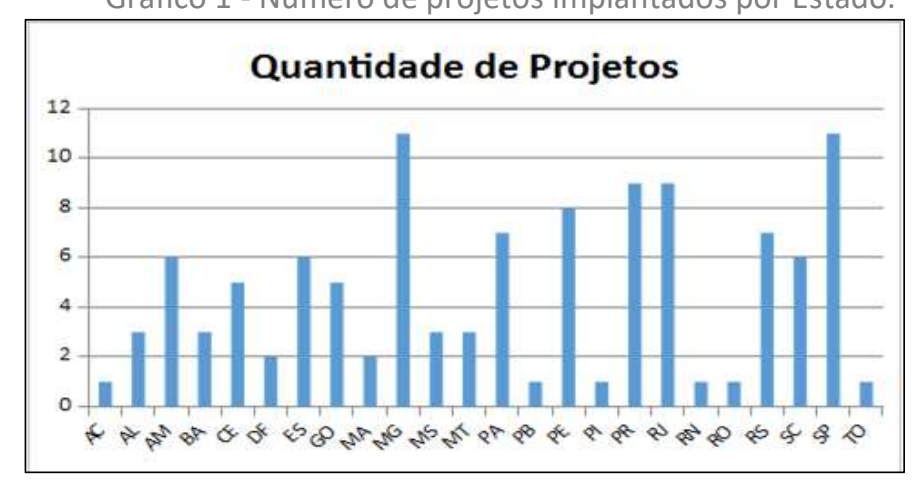

Fonte: dados da pesquisa (2017)

Cabe destacar que o Gráfico 1 contempla todos os Estados brasileiros, onde foi desenvolvido algum projeto de Tecnologia Social, a região Sudeste teve um destaque maior com relação às outras regiões com 37 projetos certificados.

Já a região Centro-Oeste, foi a que teve o menor número de projetos implementados, 11 ao todo, num universo de 112 encontrados, pois alguma 
tecnologia foi implementada em mais de um Estado. Vale ressaltar que, foram analisados somente projetos que tinham relação ao contexto da questão ambiental em seu escopo.

Na Tabela 3, é apresentado o ano de certificação das Tecnologias Sociais analisadas.

Tabela 3 - Ano de certificação de cada tecnologia.

\begin{tabular}{|c|c|c|}
\hline Ano da certificação & Quantidade por Ano & Porcentagem \% \\
\hline 2001 & 1 & $1,3 \%$ \\
\hline 2003 & 2 & $2,6 \%$ \\
\hline 2005 & 1 & $1,3 \%$ \\
\hline 2007 & 2 & $2,6 \%$ \\
\hline 2009 & 3 & $3,8 \%$ \\
\hline 2011 & 20 & $25,6 \%$ \\
\hline 2013 & 10 & $12,8 \%$ \\
\hline 2015 & 12 & $15,4 \%$ \\
\hline 2017 & 16 & $20,5 \%$ \\
\hline Não informado & 11 & $14,1 \%$ \\
\hline Total & 78 & $100 \%$ \\
\hline
\end{tabular}

Fonte: dados da pesquisa (2017)

Destaca-se, ainda, que alguns projetos de Tecnologia Social da amostra selecionada, não tiveram a sua certificação junto ao Banco de Tecnologias da Fundação Banco do Brasil, visto que, isso leva algum tempo até que passem por todo o processo de reconhecimento que a caracterize com uma Tecnologia Social.

Como pode ser observado na Tabela 3, o ano de 2011, teve um destaque maior com relação aos outros anos, pois foi o ano com o maior número de certificações, 20 certificações, seguido por 2017 com 16, 2015 com 12, 2013 com 10, 2009 com 3, 2003 e 2007 com 2 cada ano e 2001 e 2005 com apenas 1 certificação cada.

Na Tabela 4, apresenta-se o público-alvo das Tecnologias Sociais.

Tabela 4 - Público-alvo das Tecnologias Sociais.

\begin{tabular}{|l|c|c|}
\hline \multicolumn{1}{|c|}{ Público-alvo } & Número & Porcentagem \% \\
\hline Agricultores & 54 & $20,93 \%$ \\
\hline Crianças/Jovens/Adultos e Idosos & 35 & $13,57 \%$ \\
\hline Alunos & 29 & $11,24 \%$ \\
\hline Famílias de baixa renda & 20 & $7,75 \%$ \\
\hline Lideranças Comunitárias & 16 & $6,20 \%$ \\
\hline População Ribeirinha/Pescadores & 13 & $5,04 \%$ \\
\hline Mulheres & 12 & $4,65 \%$ \\
\hline População em Geral & 12 & $4,65 \%$ \\
\hline Povos Tradicionais/Povos Indígenas & 11 & $4,26 \%$ \\
\hline Professores & 11 & $4,26 \%$ \\
\hline Assentados Rurais & 8 & $3,10 \%$ \\
\hline Desempregados & 6 & $2,33 \%$ \\
\hline Organização não governamental & 5 & $1,94 \%$ \\
\hline Empreendedores & 5 & $1,94 \%$ \\
\hline Catadores de Material Reciclável & 4 & $1,55 \%$ \\
\hline
\end{tabular}




\begin{tabular}{|l|c|c|}
\hline Artesãos & 4 & $1,55 \%$ \\
\hline Gestores Públicos & 3 & $1,16 \%$ \\
\hline Outros & 3 & $1,16 \%$ \\
\hline População em Situação de Rua & 2 & $0,78 \%$ \\
\hline Afrodescendentes/Quilombolas & 2 & $0,78 \%$ \\
\hline Analfabeto & 1 & $0,39 \%$ \\
\hline Grupos de minorias e em situação de exclusão & 1 & $0,39 \%$ \\
\hline $\begin{array}{l}\text { Participantes do grupo das comunidades } \\
\text { escolhidas }\end{array}$ & 1 & $0,39 \%$ \\
\hline Total & $\mathbf{2 5 8}$ & $\mathbf{1 0 0 \%}$ \\
\hline
\end{tabular}

Fonte: dados da pesquisa (2017)

Cabe evidenciar, que alguns projetos de Tecnologia Social da amostra selecionada junto ao Banco de Tecnologias da Fundação do Banco do Brasil contemplam mais de um tipo de público-alvo, conforme apresenta a Tabela 4.

Foram identificados os seguintes públicos-alvo: agricultores (54 projetos); crianças/jovens/adultos e idosos (35 projetos); alunos (29 projetos); famílias de baixa renda (20 projetos); lideranças comunitárias (16 projetos); população Ribeirinha e pescadores (13 projetos); mulheres e população em geral com (12 projetos) cada; povos tradicionais, povos Indígenas e professores (11 projetos) cada; assentados rurais (8 projetos); desempregados (6 projetos); organização não governamental e empreendedores com (5 projetos) cada; catadores de material reciclável e artesãos com (4 projetos) cada; gestores públicos e outros com (3 projetos) cada; população em situação de rua, afrodescendentes e quilombolas com ( 2 projetos) cada; analfabeto, grupos de minorias e em situação de exclusão e participantes do grupo das comunidades escolhidas com (1 projeto) cada, totalizando 258 projetos.

Na Tabela 5, é apresentado os Problemas envolvidos com Tecnologias Sociais

Tabela 5 - Problemas envolvidos nas Tecnologias Sociais.

\begin{tabular}{|l|c|c|}
\hline Problemática levantada & Quantidade & $71,8 \%$ \\
\hline Meio Ambiente & 6 & $7,7 \%$ \\
\hline Renda & 2 & $2,6 \%$ \\
\hline Recursos Hídricos & 4 & $5,2 \%$ \\
\hline Alimentação & 7 & $9,0 \%$ \\
\hline Educação & 2 & $2,6 \%$ \\
\hline Saúde & 1 & 1,35 \\
\hline Energia & 78 & $100 \%$ \\
\hline Total & & \\
\hline
\end{tabular}

Fonte: dados da pesquisa (2017) 
Pode-se observar na Tabela 5, as problemáticas levantadas, que foram delineadas como: Meio Ambiente, Renda, Recursos Hídricos, Alimentação, Educação, Saúde e Energia. Sendo encontradas, 78 Tecnologias Sociais relacionadas a problemática da pesquisa (Meio Ambiente).

Na problemática do Meio Ambiente, o foco principal foi buscar meios ou alternativas que pudessem amenizar os impactos que esse tema vem sofrendo ao longo dos anos, causado por exemplo, pelo surgimento de grandes cidades e indústrias, entre outros fatores, que acabam causando um grande desequilíbrio ao Meio Ambiente e tornando escassos seus recursos naturais.

Apesar das outras temáticas abordarem temas mais específicos como: renda, energia, saúde, educação, alimentação e recursos hídricos, a questão ambiental também foi levada em conta. Afinal, a matéria (produto) base para desenvolvimento dessas tecnologias estão ligadas diretamente com o meio ambiente.

Para que possam ter esses recursos a sua disposição, é preciso que o desenvolvimento dessas Tecnologias Sociais esteja voltado para um desenvolvimento sustentável e equilibrado do Meio Ambiente ao seu redor, e para que isso ocorra, é necessária uma integração de toda a comunidade local, para que possam assimilar e disseminar para toda a comunidade essa tecnologia apropriada.

\section{CONSIDERAÇÕES FINAIS}

Considerando a pertinência da discussão do impacto da ação humana, por meio do emprego de tecnologias, no Meio Ambiente, o objetivo desse trabalho foi de analisar junto ao Banco de Tecnologias Sociais da Fundação Banco do Brasil, quais tecnologias estavam relacionadas a questão ambiental, uma vez que, tratase de uma temática que atualmente está no centro de muitos debates, tanto na esfera política, como acadêmica, nas escolas, no meio empresarial, entre outros.

Com base no contexto analisado, pode-se mapear que o número de projetos encontrados na área ambiental ainda é relativamente baixa em comparação ao total de trabalhos registrados no banco de dados, apenas 78 de um universo de 1011.

Pode-se ainda vislumbrar que, nos anos de 2008 e 2009, teve um aumento significativo no número de implantação de Tecnologias Sociais, tendo como objetivo principal a questão da preservação das nascentes de água.

Por meio dos dados analisados, vislumbrou-se que aliada a preocupação com - Meio Ambiente, a necessidade de geração de renda, educação, recursos hídricos, saúde, entre outras, foram temáticas que despertaram a maior atenção para com as Tecnologias Sociais pesquisadas.

Embora o percentual de tecnologias voltadas para questão ambiental seja pequeno, 78 de 1011, totalizando 7,7\% do total, essas tecnologias auxiliaram na emancipação das comunidades que foram beneficiadas pela sua implementação, promovendo a inclusão social, a diminuição da fronteira entre as classes sociais, melhora da qualidade de vida de toda a comunidade envolvida, gerando renda e alimentos. 
Portanto, conforme analisado, a Tecnologia Social vem auxiliar em diversos contextos com intuito de possibilitar uma melhora significativa no que diz respeito às condições de vida das pessoas que são beneficiadas diretamente ou indiretamente por essa tecnologia, seja um pequeno grupo ou até mesmo uma comunidade inteira. Devido a sua capacidade de interação com toda a comunidade envolvida, e a possibilidades de contextualizar e ser adaptada a realidade de cada comunidade, tem envolvido em muitos casos, diversas áreas como: Saúde, Educação, Energia, Meio Ambiente, entre outras, conforme pode ser observado no mapeamento realizado.

Espera-se que os presentes achados possam contribuir para o debate acerca da relação entre Tecnologias e Meio Ambiente, assunto de extrema importância para o contexto em que vivemos e para a própria sobrevivência da humanidade. 


\title{
Environmental practices under the social technology perspective
}

\begin{abstract}
The Social Technology has contributed in the attempt to minimize many of the social problems not taken care of by the public sphere, in a special way related to the problematic of the Environment. Thus, this paper aimed to analyze the Social Technology projects that present solutions to environmental issues. To do so, as a methodological approach, a qualitative was used, and the data collected in the Bank of Social Technologies of the Banco do Brasil Foundation. As a result, 78 projects out of a total of 1011 registered in the analyzed database were identified, these technologies can help in the emancipation of the communities that benefit from its implementation, promoting social inclusion, reducing the boundary between social classes, and improving the quality of life of those involved. Furthermore, it was found that the years of 2008 and 2009 were the periods of greater implementation of projects and the main public consists of farmers.
\end{abstract}

KEYWORDS: Environment. Social Technologies. Technological Alternatives. 


\section{REFERÊNCIAS}

BAUMGARTEN, M. Tecnologias sociais e inovação social. 2006. Disponível em: www.projeccia.com.br/images/download/.../Tecnologias-sociais-einovacao-social.pdf. Acesso em: 30/10/2017.

BAVA, S. C. Tecnologia social e desenvolvimento local. Tecnologia social: uma estratégia para o desenvolvimento. Rio de Janeiro: FBB, p. 103 -116, 2004.

CONAMA, Resolução №. 306 de 05 de julho de 2002. Estabelece os requisitos mínimos e o termo de referência para realização de auditorias ambientais, 2002. Disponível em: http://www.mma.gov.br/port/conama/legiabre.cfm?codlegi=306. Acesso em: 28/10/2017.

COSTA, A. B. (org.) Tecnologia Social e Políticas Públicas. São Paulo: Instituto Pólis; Brasília: Fundação Banco do Brasil, 2013.

DAGNINO, R.; NOVAES, H. T. A Adequação Sócio-técnica como insumo para a recuperação dos Institutos Públicos de Pesquisa. Revista Brasileira de Gestão e Desenvolvimento Regional, v. 1, n. 3, 2004. Disponível em: http://www.rbgdr.net/revista/index.php/rbgdr/article/view/60. Acesso em: 01/11/2017.

DE MAGALHÃES, S. B. et al. Tecendo Inovação Social no Paraná. FIEP-Federação das Indústrias do Estado do Paraná, 2 p. 145, 2008. Disponível em: http://www.sistemafiep.org.br/uploadAddress/volumedois[36097][747 13]. pdf\#page=139. Acesso em: 25/10/2017.

DUQUE, T. O.; VALADÃO, J. A. D. Abordagens teóricas de tecnologia social no Brasil. Revista Pensamento Contemporâneo em Administração, v. 11, n. 5, p. 1-19, 2017.

FEENBERG, A. Cinco paradoxos da tecnologia e da política de desenvolvimento. OTERLOO, A. et al. Tecnologias Sociais: caminhos para a sustentabilidade. Brasília: s/n, p. 99 -116, 2009.

FUNDAÇÃO BANCO DO BRASIL. Banco de Tecnologias Sociais. Disponível em: http://tecnologiasocial.fbb.org.br/tecnologiasocial/principal.htm. Acesso em: 11/10/2017.

GARCIA, S. G. A tecnologia social como alternativa para a reorientação da economia. Estudos Avançados., São Paulo, v. 28, n. 82, p. 251-275, Dec. 2014. Disponível em: http://www.scielo.br/scielo.php?script=sci_arttext\&pid=S010340142014000300015\&Ing=en\&nrm=iso. Acesso em: 09/08/2017.

GAPINSKI, E. F. P. et al. Prática tecnológica e tecnologia social: um estudo a partir dos pressupostos teóricos da construção social da tecnologia. Revista Tecnologia e Sociedade, v. 14, n. 30, 2018. 
GIL, . C. Como elaborar projetos de pesquisa. $5^{a}$ edição, Editora atlas. 2010.

LASSANCE JR. A. E.; PEDREIRA, J. S. Sobre o Marco Analíticoconceitual da Tecnologia Social. In: FBB. Tecnologia Social: uma estratégia para o desenvolvimento. Fundação Banco do Brasil, Rio de Janeiro, p. 65- 70, 2004.

LUNARDI, G. M. Tecnologias Inclusivas e Inovação Social, p. 61. Araranguá, $2015 . \quad$ Disponível em: https://publicacoes.rexlab.ufsc.br/index.php/sppi/article/view/31/10. Acesso em: 30/10/2017.

OLIVEIRA, L. D. A ideologia do desenvolvimento sustentável: notas para reflexão. Revista Tamoios, v. 1, n. 2, 2010.

PEREIRA, L. C. B.; FREITAS, C. C. G. Educação na tecnologia social: análise de experiências. Revista Tecnologia e Sociedade, v. 14 , n. 30, 2018.

REIS, L. B.; FADIGAS, E. A. A. Energia, Recursos Naturais e a Prática do Desenvolvimento Sustentável. 2. ed. Barueri: Manole, 2012.

VALADÃO, J. A. D.; DE ANDRADE, J. A.; CORDEIRO NETO, J. R.. Abordagens sociotécnicas e os estudos em tecnologia social. Revista Pretexto, v. 15, n. 1, p. 44-61, 2014.

WEISS, Z. Tecnologia Social: Os Desafios de uma Abordagem Holística. Revista Tecnologias Sociais: Caminhos para a sustentabilidade, p.166-172. Brasília, 2009. Disponível em: file:///C:/Users/teste/Downloads/rts_caminhos.pdf. Acesso em: 22/10/2017.

Recebido: 05 mai 2018 .

Aprovado: 12 dez 2018

DOI: 10.3895/rts.v15n38.8259

Como citar: OLIVEIRA, S.B.; et. al. Práticas ambientais sob a perspectiva da tecnologia social. Revista

Tecnologia e Sociedade, Curitiba, v. 15, n. 38, p. 75-89, out/dez. 2019. Disponível em:

https://periodicos.utfpr.edu.br/rts/article/view/8259 Acesso em: XXX

Correspondência:

Direito autoral: Este artigo está licenciado sob os termos da Licença Creative Commons-Atribuição 4.0 Internacional 